\title{
Masreliez-Equalized VOFDM in Non-Gaussian Channels: Power Line Communication Systems
}

\author{
Cinna Soltanpur, Member, IEEE, Khaled M. Rabie, Member, IEEE, Bamidele Adebisi, Senior Member, IEEE and \\ Andrew Wells, Member, IEEE,
}

\begin{abstract}
In this paper, we derive a non-linear equalizer for a fading channel with non-Gaussian noise. In particular, we look into the effects of non-Gaussian noise over power line channels that severely affect communication signals. Unlike most existing work based on orthogonal frequency-division multiplexing (OFDM) in the literature, we investigate the performance of vector OFDM (VOFDM) over multi-path power line communication (PLC) channels contaminated with Middleton Class-A noise. To reduce the impact of impulsive noise we propose a novel filter to equalize the output of the channel. The performance of the equalizer is evaluated in terms of bit error rate (BER), and the impact of several impulsive noise parameters are examined at the receiver. Results show that the proposed system can considerably improve the BER performance in comparison to the conventional OFDM scheme. In addition, it is shown that increasing the number of vector blocks of the VOFDM system will enhance the BER performance under the same condition. The proposed nonlinear equalizer improves the performance of VOFDM system successfully at low signal-to-noise ratios (SNRs), at some instances it nearly halved the probability of error with respect to linear filter.
\end{abstract}

Index Terms-Middleton Class-A noise, noise mitigation, power line communication (PLC), vector orthogonal frequencydivision multiplexing (VOFDM).

\section{INTRODUCTION}

$\mathbf{T}$ HE existing infrastructure of the power line communication (PLC) network makes a good case for the development of many smart grid and home networking applications [1], [2]. In fact, PLC is increasingly becoming a promising alternative solution to wireless and optical communications in networking with the advantage of pre-existing wiring installations, or regions where access to wireless communications is scant.

There are several characteristics which affect the performance of PLC communication systems, for the power lines were originally designed for $50-60 \mathrm{~Hz}$ electrical power distribution. Communication signals at high frequencies over the power cables can severely be affected as attenuation increases exponentially with both frequency and distance. Communication signals also suffer from multi-path fading resulting in signal distortion and inter-symbol interference (ISI). However, the most impeding factor is the presence of

Cinna Soltanpur is with the School of Electrical and Computer Engineering, University of Oklahoma, Norman, OK 73019 USA. (e-mail: cinna@ou.edu).

Khaled M. Rabie and Bamidele Adebisi are with the School of Electrical Engineering, Manchester Metropolitan University, Manchester, M15 6BH, UK. (e-mails: k.rabie@mmu.ac.uk; b.adebisi@mmu.ac.uk).

Andrew Wells is with the Jaguar Land Rover Limited, Gaydon, Warwick, CV35 ORR, UK. (e-mail: awells@jaguarlandrover.com). impulsive noise which is the dominant element of channel noise and can have power spectral density of up to $50 \mathrm{~dB}$ above the background noise [3], [4]. Middleton Class-A noise model is a widely used model for PLC channels and will be adopted in this paper [5], [6]. Generally, multi-carrier modulation systems, such as orthogonal frequency-division multiplexing (OFDM), are more resistant to impulsive noise than singlecarrier systems [7]; for this reason, OFDM has been the main modulation technique for most PLC standards both in narrowband and broadband technologies [8]-[11]. In addition, error control coding schemes can further improve performance in impulsive noise channels. For example, the authors in [7] studied the performance of coded OFDM in PLC system. However, when the noise power is high, other noise mitigation techniques should be implemented along with OFDM. For instance, clipping/blanking of the incoming received signal when it exceeds a certain threshold has been used in [12]-[14]. These methods are built upon the success of simple empirical blankers and clippers.

It is known that OFDM systems suffer from spectral nulls due to ISI in the channel [15]. In addition, there are other issues with OFDM such as its sensitivity to carrier frequency offset (CFO) and high peak-to-average power ratio (PAPR) properties [16]. Vector OFDM (VOFDM) is introduced by Xia in [17] to alleviate the mentioned shortcomings of OFDM system. It is shown that VOFDM has a smaller peak-toaverage power ratio (PAPR), increases diversity order, and is less sensitive to CFO [16]. Authors in [16] have studied the performance of VOFDM system over frequency selective channel and showed that the superior bit error rate (BER) performance of VOFDM is due to signal space diversity. Furthermore, the guard band configuration, synchronization and turbo-equalization are discussed in [18].

This paper studies the VOFDM system over a multi-path power line channel in the presence of Middleton ClassA noise. The main motivation for proposing VOFDM for PLC resides in its ability to adapt to frequency selective fading and its low PAPR features. In order to reduce the impact of impulsive noise, we propose a non-linear equalizer, specifically the Masreliez filter ${ }^{1}$, at the receiver for complex valued estimation in non-Gaussian environment. Therefore the main contributions of this paper are as follows: First, we propose an explicit non-linear filter for the impulsive noise

\footnotetext{
${ }^{1}$ Kalman filter for non-Gaussian noise introduced by Masreliez in [19], is an efficient algorithm for estimating a linear stochastic system with low computational burden. However, the Kalman equalizer is known to have a poor BER performance [20].
} 

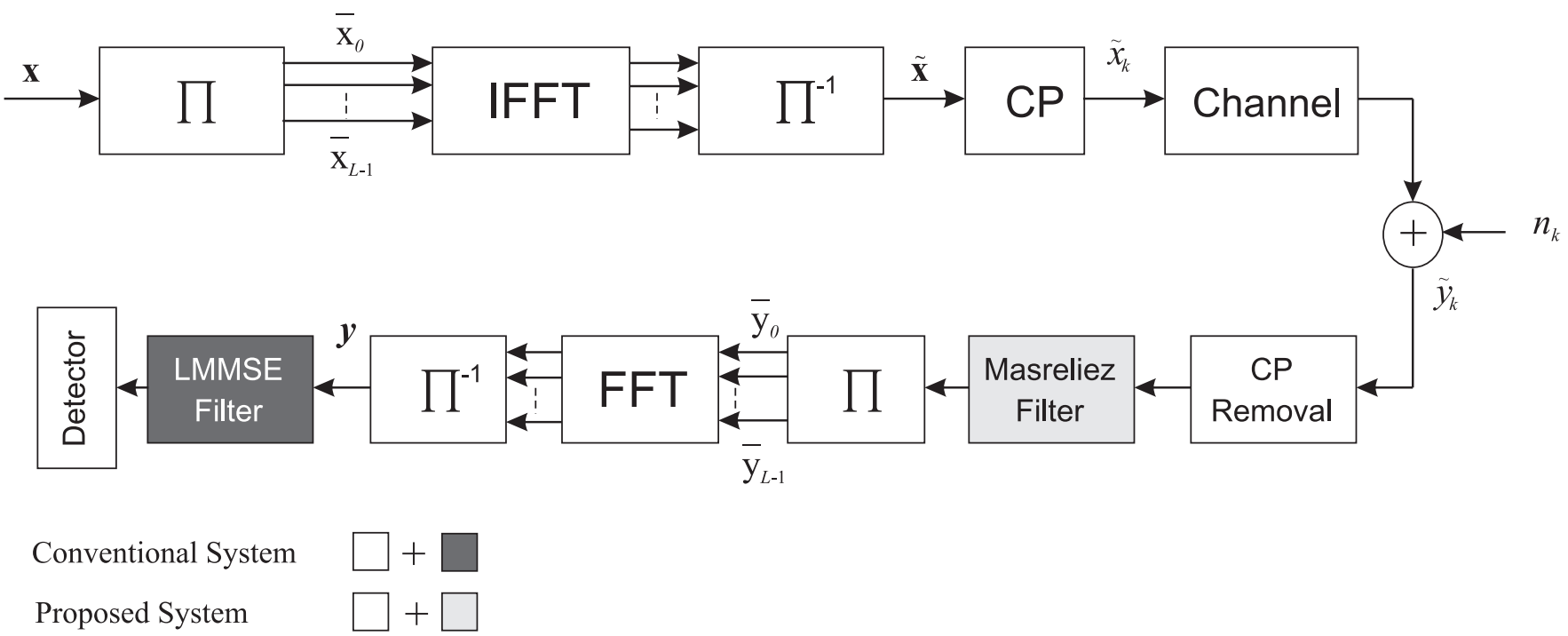

Fig. 1. The block diagram of the proposed and conventional VOFDM systems.

PLC channel and compare its performance to that of the linear equalizer. The second contribution resides in examining the impact of different VOFDM system parameters and noise scenarios on the performance of the proposed system. It is worth mentioning that at the limit when the magnitude of spikes are much higher than background noise, the Masreliez filter reduces to a blanker [14].

The rest of this paper is organized as follows. In Section II, the general system model is described and VOFDM is briefly reviewed. In Section III, derivations of linear minimum mean square error (LMMSE) and Masreliez equalizer are given. Section IV presents the PLC channel and evaluates the performance of the proposed system over PLC channels. After that, the simulation results are presented and discussed. Finally, Section V concludes the paper and outlines the main results.

The notations used in this paper are as follows: Bold uppercase and bold lowercase letters denote matrices and vectors, respectively. Conjugate transpose is denoted by $(.)^{*}$ and $\otimes$ represents the Kroneker product. The notation $\|$.$\| represent$ Euclidean norm for vectors or absolute value of a complex number. Circularly symmetric, complex Gaussian distribution with mean $\mu$ and variance $\sigma^{2}$ is denoted by $\mathcal{C N}\left(\mu, \sigma^{2}\right)$; I is the identity matrix and diag[.] represents a block diagonal matrix whose diagonal elements are matrices; $\operatorname{det}(A)$ indicates the determinant of matrix $A$.

\section{SYSTEM MOdEL}

A basic block diagram of the system considered in this paper is shown in Fig. 1. First, a block of modulated symbols of size $N$, denoted as $\mathbf{x}=\left[x_{0}, \ldots, x_{N-1}\right]$, is rearranged as $\overline{\mathbf{x}}$ by passing it through a permutation matrix $\Pi$, and divided into $L$ blocks $\overline{\mathbf{x}}=\left[\overline{\mathbf{x}}_{0}, \ldots, \overline{\mathbf{x}}_{L-1}\right]$. Then, inverse fast Fourier transform (IFFT) of size $M$ is performed on each vector to realize the time domain symbols. After that, the resultant sequence is once more permuted using matrix $\Pi^{-1}$ to produce $\tilde{\mathbf{x}}=\left[\tilde{x}_{0}, \ldots, \tilde{x}_{N-1}\right]$. The cyclic prefix $(\mathrm{CP})$ of appropriate size is now added to the data before transmission. Given that $h_{k}, k=0, \ldots, K$, represents the channel impulse response, after the removal of the $\mathrm{CP}$, the time domain signal at the receiver can be expressed as

$$
\tilde{y}_{k}=\sum_{k=0}^{K} h_{k} \tilde{x}_{(n-k) \equiv N}+n_{k},
$$

where $n_{k}$ is the noise. The indexes of $x$ 's are chosen congruent to $0, \ldots, N-1$ modulo $N$. Next, the received signal goes through the reverse process of what performed at the transmitter side. That is, the vector $\tilde{\mathbf{y}}$ is permuted by the matrix $\Pi$ and is split into $L$ vectors as $\left[\overline{\mathbf{y}}_{0}, \ldots, \overline{\mathbf{y}}_{L-1}\right]$. Fast Fourier transform (FFT) is then performed on each vector block to retrieve the original transmitted signal, i.e., $\overline{\mathbf{x}}$, which is then permuted back to the original positions using the matrix $\Pi^{-1}$.

The channel response can be expressed in rows of equivalent matrix $\mathbf{H}$. Since $\mathbf{H}$ is a cyclic matrix, the overall procedure block diagonalizes the matrix $\mathbf{H}$ (see Sec. II.B.) Hence, we can write the output of the VOFDM system in block form as follows

$$
\mathbf{y}_{l}=\mathbf{H}_{l} \mathbf{x}_{l}+\overline{\mathbf{n}}_{l}
$$

where $\mathbf{y}_{l}, \mathbf{x}_{l}$, and $\overline{\mathbf{n}}_{l}$ are vectors each of size $M$ for $l=$ $0,1, \ldots, L-1$. The matrix $\mathbf{H}_{l}$ is a factor circulant matrix given by

$$
\mathbf{H}_{l}=\left[\begin{array}{cccc}
h_{0}^{l} & \omega h_{M-1}^{l} & \cdots & \omega h_{1}^{l} \\
h_{1}^{l} & h_{0}^{l} & \cdots & \omega h_{2}^{l} \\
\vdots & \vdots & \ddots & \vdots \\
h_{M-1}^{l} & h_{M-2}^{l} & \cdots & h_{0}^{l}
\end{array}\right]_{M \times M}
$$

where $\omega=\exp \left(-2 \pi i \frac{l}{L}\right)$. It should also be noted that $\tilde{x}$ is a weighted sum of independent random variables and for large $n$, by the central limit theorem, it has a Gaussian distribution. For further explanation of VOFDM system and performance analysis we refer the reader to [15]. 


\section{A. Block Diagonalization of $\mathbf{H}$}

In OFDM, sub-carriers are orthogonal. Indeed, the unitary discrete Fourier transform (DFT) matrices diagonalize matrix $\mathbf{H}$. In this section, we explain how the cyclic matrix $\mathbf{H}$ is block diagonalized in VOFDM. Let $\mathbf{S}$ be a block shift matrix as

$$
\mathbf{S}=\left[\begin{array}{cccc}
\mathbf{0} & I_{M} & \cdots & \mathbf{0} \\
\vdots & & \ddots & \vdots \\
\mathbf{0} & \ddots & & I_{M} \\
I_{M} & \mathbf{0} & \cdots & \mathbf{0}
\end{array}\right]_{N \times N}
$$

Then, the circulant matrix $\mathbf{H}$ can be expressed using powers of $\mathbf{S}$ as

$$
\mathbf{H}=\sum_{k=0}^{L-1} \mathbf{S}^{-k} \operatorname{diag}\left[\breve{\mathbf{H}}_{k}\right]=\mathbf{U} \sum_{k=0}^{L-1} \boldsymbol{\Lambda}^{-k} \mathbf{U}^{*} \operatorname{diag}\left[\breve{\mathbf{H}}_{k}\right]
$$

where $\breve{\mathbf{H}}_{k}$ 's are $M \times M$ matrices of the first $M$ columns of $\mathbf{H}$, and $\Lambda$ is the eigenvalues of matrix $\mathbf{S}$. Since $\operatorname{diag}\left[\breve{\mathbf{H}}_{k}\right]=$ $\mathbf{U}\left\{\operatorname{diag}\left[\breve{\mathbf{H}}_{k}\right]\right\} \mathbf{U}^{*}$, we can write

$$
\begin{aligned}
\mathbf{H} & =\mathbf{U}\left\{\sum_{k=0}^{L-1} \boldsymbol{\Lambda}^{-k} \operatorname{diag}\left[\breve{\mathbf{H}}_{k}\right]\right\} \mathbf{U}^{*} \\
& =\mathbf{U}\left\{\operatorname{diag}\left[\mathbf{H}_{0}, \ldots, \mathbf{H}_{L-1}\right]\right\} \mathbf{U}^{*},
\end{aligned}
$$

where $\mathbf{H}_{i}, i=1,2, \ldots, L$, is given in (3).

It is clear that $\mathbf{S}$ can be looked at as the Kronecker product of shift matrix $\mathbf{S}_{L}$ of size $L \times L$ and the identity matrix $\mathbf{I}_{M}$ for $N=L M$. The eigenvalues and eigenvectors of the matrix $\mathbf{S}_{L} \otimes \mathbf{I}_{M}$ are the set of all the $\lambda_{j} \bar{\lambda}_{k}$ and the eigenvectors $\mathbf{v}_{j} \otimes \mathbf{p}_{k}$, where $\lambda_{j}$ and $\mathbf{v}_{j}$ are the eigenvalues and eigenvectors of $\mathbf{S}_{L}$ whereas $\bar{\lambda}_{k}$ and $\mathbf{p}_{k}$ are the eigenvalues and eigenvectors of $\mathbf{I}_{M}$ [21]. We further note that the eigenvector matrix of $\mathbf{S}_{L}$ is a DFT matrix of size $L \times L$ and the eigenvector matrix of $\mathbf{I}_{M}$ is the identity matrix of size $M \times M$. Hence, the eigenvector matrix $\mathbf{U}$ can be written as $\mathbf{U}=\mathbf{D}_{L} \otimes \mathbf{I}_{M}$ (this is justified by [21, Thm 13.10] and $\mathbf{S}_{L} \otimes \mathbf{I}_{M}$ being a normal matrix). Therefore, there exists a permutation matrix $\Pi$ such that [22]

$$
\mathbf{D}_{L} \otimes \mathbf{I}_{M}=\boldsymbol{\Pi}^{-1}\left(\mathbf{I}_{M} \otimes \mathbf{D}_{L}\right) \boldsymbol{\Pi} .
$$

Since $\mathbf{D}_{L}$ is a DFT matrix, $\mathbf{I}_{M} \otimes \mathbf{D}_{L}$ is a block diagonal matrix of $L$ DFT matrices of size $L$. In our derivation, it is shown that the identity matrix $\mathbf{I}_{M}$ can be replaced with any other diagonal matrix. The representation in (7) helps us to use FFT, which is practically a computationally efficient method, on each block of data. It should also be highlighted that the permutation matrix $\Pi$ is only a rearrangement of a vector of input data into a matricial form where the FFT/IFFT is performed on each row. Furthermore, it can be observed that when $M=1$, the matrix $\mathbf{U}$ is simply a DFT matrix of size $N$ and the VOFDM system reduces to conventional OFDM.

One more remark before moving onto the detection section is that the matrix $\mathbf{H}_{l}$ is basically a factor circulant matrix and can be diagonalized as $\overline{\mathbf{H}}_{l}=\mathbf{U}_{l}^{*} \mathbf{H}_{l} \mathbf{U}_{l}$, where the elements of matrix $\mathbf{U}_{l}$ is given explicitly as [15]

$$
\left[\mathbf{U}_{l}\right]_{r, s}=\frac{1}{\sqrt{M}} \exp \left(-2 \pi i \frac{(l+r L) s}{N}\right),
$$

where $r$ and $s \in\{0,1, \ldots M-1\}$.

\section{Detection StRategies}

In this section, we first derive the LMMSE equalizer for Gaussian source, and the equalizer is then generalized for nonGaussian noise. We discuss the Masreliez equalizer which is employed to combat the degradation impact of impulsive noise channels. Note that with the Gaussian assumption, we can write the optimum maximum likelihood (ML) detection for VOFDM system as [15]

$$
\hat{\mathbf{x}}_{l}^{M L}=\arg \min _{\mathbf{x} \in \mathbb{X}^{M}}\left\|\mathbf{y}_{l}-\mathbf{H}_{l} \mathbf{x}_{l}\right\|^{2},
$$

where $\mathbb{X}$ is the set of input alphabets and $\mathbb{X}^{M}$ is the $M$-th Cartesian product. It is evident that the complexity of ML detection grows exponentially with respect to the size of vector $\mathbf{x}_{l}$ as well as the modulation order. The equalizer provides a less complex solution to the detection problem [15].

\section{A. LMMSE: Frequency Domain Equalization}

Minimum mean square error (MMSE) and other equalizers have already been proposed for VOFDM in [15]. The main advantage of using an equalizer for detection is the reduced computational complexity with respect to ML detector [15]. In this technique, the symbol-by-symbol detection is done on the equalizer output and the soft values can be calculated as required. When MMSE is complicated, we settle for the best linear estimator, i.e., LMMSE. These two equalizers are exactly the same for Gaussian noise. In this paper we derive the LMMSE estimator for VOFDM.

Now, assuming that the matrices $\boldsymbol{\Sigma}_{x}$ and $\boldsymbol{\Sigma}_{v}$ are the covariance matrices of the input symbols and noise, respectively, we can express the weight matrix for LMMSE filter in time domain as [23], [24]

$$
\mathbf{K}_{l}^{L M M S E}=\Sigma_{x} \mathbf{H}_{l}^{*}\left(\mathbf{H}_{l} \Sigma_{x} \mathbf{H}_{l}^{*}+\boldsymbol{\Sigma}_{n}\right)^{-1} .
$$

and the LMMSE estimate of input vector of the symbols $\hat{\mathbf{x}}_{l}$ can be written as

$$
\hat{\mathbf{x}}_{l}^{L M M S E}=\mathbf{K}_{l}^{L M M S E}\left(\mathbf{H}_{l} \mathbf{x}_{l}+\mathbf{U}_{l} \mathbf{n}_{l}\right) .
$$

However, we know from (8) that the factor-circulant matrix $\mathbf{H}_{l}$ can be diagonalized and therefore (11) can be expressed as

$$
\begin{aligned}
\hat{\mathbf{x}}_{l}^{L M M S E}=\mathbf{U}_{l}^{*} \overline{\mathbf{H}}_{l}^{*}\left(\overline{\mathbf{H}}_{l} \overline{\mathbf{H}}_{l}^{*}+\mathbf{U}_{l} \boldsymbol{\Sigma}_{n} \mathbf{U}_{l}^{*}\right)^{-1} & \\
& \overline{\mathbf{H}}_{l} \mathbf{U}_{l} \mathbf{x}_{l}+\mathbf{K}^{L M M S E} \mathbf{U}_{l} \mathbf{n}_{l} .
\end{aligned}
$$

In diagonal form, (12) can be further simplified to

$$
\begin{array}{r}
\hat{\mathbf{x}}_{l}^{L M M S E}=\mathbf{U}_{l}^{*} \operatorname{diag}\left\{\frac{\left|\bar{h}_{0}^{l}\right|^{2}}{\left|\bar{h}_{0}^{l}\right|^{2}+\rho^{-1}}, . .\right. \\
\left.. ., \frac{\left|\bar{h}_{M-1}^{l}\right|^{2}}{\left|\bar{h}_{M-1}^{l}\right|^{2}+\rho^{-1}}\right\} \mathbf{U}_{l} \mathbf{x}_{l}+\mathbf{K}_{l}^{L M M S E} \mathbf{U}_{l} \mathbf{n}_{l} .
\end{array}
$$


As mentioned earlier, comparing (13) and [15, Eq. 9], it can be observed that LMMSE and MMSE estimators will have the same representation for the case of Gaussian noise.

Now, to find the hard decisions we use the following argument

$$
\bar{x}_{n}^{L M M S E}=\arg \min _{x_{n} \in \mathcal{X}}\left\|\hat{x}_{n}^{L M M S E}-K_{n} x_{n}\right\|^{2},
$$

where $\bar{x}_{n}$ is the hard decision on input alphabets and $K_{n}$ is the scale factor of symbol $x_{l M+m}$ which can be calculated from $\mathbf{K}_{l}^{L M M S E}$ as [15]

$$
K_{l M+m}=\frac{1}{M} \sum_{i=0}^{M L}\left(\frac{\left|\bar{h}_{i}^{l}\right|^{2}}{\left|\bar{h}_{i}^{l}\right|^{2}+\rho^{-1}}\right) .
$$

When the input data is coded, it is essential to provide soft values to the decoder. The log-likelihood ratio (LLR) values of the LMMSE equalizer can be calculated as discussed in [25]. The extrinsic information for binary constellations are

$$
L_{e}^{a_{q}}\left(x_{n} \mid \mathbf{y}_{l}\right)=\ln \frac{p\left(x_{n}=+1 \mid \mathbf{y}_{l}\right)}{p\left(x_{n}=-1 \mid \mathbf{y}_{l}\right)}=4 \frac{R e\left\{\hat{x}_{j}\right\}}{\hat{\sigma}_{x_{j}}} .
$$

It is worth mentioning that the generalization for non-binary constellations can be found in [25].

\section{B. Non-linear Equalization}

Our aim in this section is to develop an equalizer for impulsive noise over PLC channel. First, we derive a nonlinear equalizer for the general non-Gaussian case. Following the same procedure as in [19], we can write an estimate of $\mathbf{x}$ as

$$
\hat{\mathbf{x}}^{\text {Masreliez }}=\mathbf{U}^{*} \Sigma_{\tilde{x}} \mathbf{H}^{*} \frac{\partial}{\partial \tilde{\mathbf{y}}} \ln p(\tilde{\mathbf{y}}) .
$$

where $p(\tilde{\mathbf{y}})$ is the probability of observing vector $\tilde{\mathbf{y}}$ at the output in a non-Gaussian noise environment.

Proof: The proof is given in Appendix A.

This is similar to what is used in [20] and [26]. Also, by replacing the noise probability with a Gaussian distribution, it is easy to verify that (17) reduces to LMMSE filter given in Sec. III-A.

To understand the equalizer in more details, we consider (17) for independent noise elements and a channel with no memory in which the size of vector $\mathbf{h}$ is 1 . We let the probability $p_{\Omega}$ indicate the noise distribution. In the calculation of $p(\tilde{\mathbf{y}})$, the integral and product are interchangeable and we can write

$$
\begin{aligned}
p(\tilde{\mathbf{y}}) & =\int \prod_{k=1}^{N} p_{\boldsymbol{\Omega}}\left(\tilde{y}_{k}-h_{1} \tilde{x}_{k}\right) p(\tilde{\mathbf{x}}) d \tilde{\mathbf{x}} \\
& =\prod_{k=1}^{N} \int p_{\boldsymbol{\Omega}}\left(\tilde{y}_{k}-h_{1} \tilde{x}_{k}\right) p\left(\tilde{x}_{k}\right) d \tilde{x}_{k}
\end{aligned}
$$

where we assumed that the elements of vector $\tilde{\mathbf{x}}_{k}$ are independent. In (38), the marginal distribution of the output values unravels; each $p\left(\tilde{y}_{k}\right)$ can be calculated separately. Clearly, the first derivative of $p\left(\tilde{y}_{k}\right)$ is a nonlinear function. Therefore, given the distribution of output values $p\left(\tilde{y}_{k}\right)$, Masreliez filter can be calculated.

The nonlinear gain function of Masreliez filter, i.e, $\partial \ln p(\tilde{y}) / \partial \tilde{y}$, is shown for different noise distributions in Fig. 2 for real values of $\tilde{y}$. In this figure, the nonlinear filter corrects the gain function around impulses mean value, i.e, $\tilde{y}=-1$. Note that, unlike the Gaussian case, the nonlinear filter is nonzero for $\tilde{y}=0$. For complex values of $\tilde{y}$ the filter is shown for impulsive noise in a three dimension plot in Fig. 3 where $x$ and $y$ axis represent the real and imaginary parts of received signal.

Next, we consider the more general case with a multitap channel in the presence of impulsive noise over PLC. In this case, the formulation does not comply to a closed form. The filter can be further simplified in different manners depending on the severity of the impulsive noise. We give an approximation of Masreliez filter by exploiting the matrix splitting method [27] for ISI channels.

Approximation by Splitting the Covariance Matrix: In this section, we derive an approximate Masreliez filter for impulsive noise. Throughout this section the assumption is that $\mathbf{H H}^{*}$ is diagonally dominant to obtain a closed form solution. Note that the impulsive noise model can be represented by hidden Markov states [28]. Meanwhile, let us assume the locations of noise impulses are known, that is to say the variance of noise is revealed for each received symbol. Let $\Sigma_{\mathbf{w}}$ be the sum of $\sigma_{i}^{2} \mathbf{J}_{i}$ where the main entries of diagonal matrix $\mathbf{J}_{i}$ are 1 when the variance of noise is $\sigma_{i}^{2}$ and 0 otherwise. Given that current state of noise characteristics $\mathbf{w}$ is known, we can write the probability of noise as follows

$$
p_{\Omega \mid W} \sim\left(2 \pi \operatorname{det} \Sigma_{\mathbf{w}}\right)^{-N / 2} \exp \left((\tilde{\mathbf{y}}-\mathbf{H} \tilde{\mathbf{x}})^{*} \Sigma_{\mathbf{w}}^{-1}(\tilde{\mathbf{y}}-\mathbf{H} \tilde{\mathbf{x}})\right) .
$$

Solving the marginal distribution of the following

$$
p_{\Omega \mid W}(\tilde{\mathbf{y}} \mid \mathbf{W}=\mathbf{w})=\int p_{\boldsymbol{\Omega} \mid \mathbf{W}}(\tilde{\mathbf{y}}-\mathbf{H} \tilde{\mathbf{x}}) p(\tilde{\mathbf{x}}) d \tilde{\mathbf{x}}
$$

we can write

$$
\begin{aligned}
p_{\Omega \mid W}(\tilde{\mathbf{y}} \mid \mathbf{W}=\mathbf{w})= & \frac{1}{\operatorname{det}\left(\frac{1}{\sigma_{x}^{2}}\left(\mathbf{H H}^{*}\right)^{-1}+\mathbf{\Sigma}_{\mathbf{w}}\right)} \\
& \times \frac{\operatorname{det} \mathbf{H}^{-1}}{\left(2 \pi \sigma_{x}^{2}\right)^{M}} \exp \left(-\tilde{\mathbf{y}}^{*} \Sigma^{-1} \tilde{\mathbf{y}}\right) .
\end{aligned}
$$

where $\Sigma^{-1}=\left(\Sigma_{\mathbf{w}}+\sigma_{x}^{2} \mathbf{H H}^{*}\right)^{-1}$. Next, we split the inverse of the covariance matrix as explained in [27]. A two term splitting with $\sigma_{1}, \sigma_{2}$, where $\sigma_{2}>\sigma_{1}$ is used. Generalization to more terms in $\Sigma_{\mathbf{w}}$ is cumbersome but straightforward. Let us define two matrices $\mathbf{C}, \mathbf{V}$ as

$$
\mathbf{C}=\left(\sigma_{x}^{2} \mathbf{H H}^{*}+\sigma_{1}^{2} \mathbf{J}_{1}-\alpha \mathbf{I}\right), \mathbf{V}=\left(\sigma_{2}^{2} \mathbf{J}_{2}+\alpha \mathbf{I}\right),
$$

which enables us to write the approximation of the inverse of the covariance matrix as 


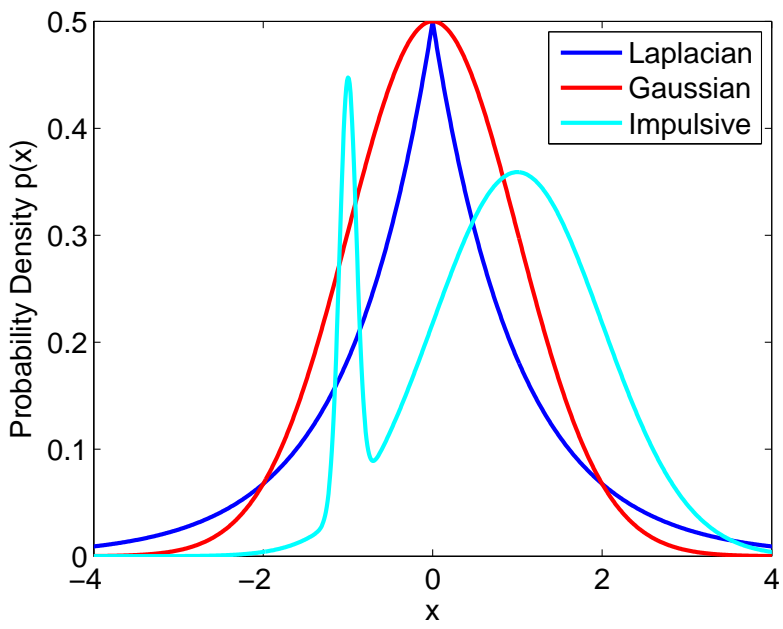

(a) Noise distributions

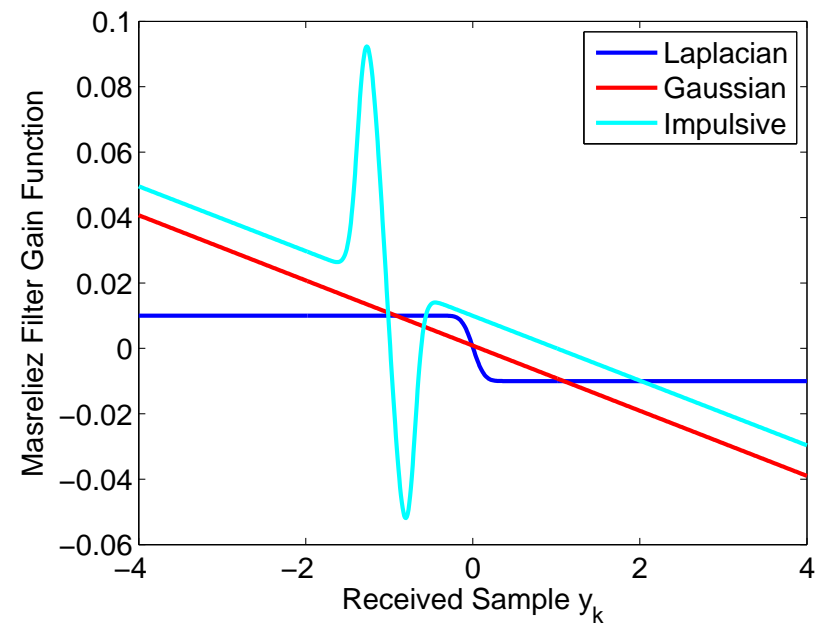

(b) Masreliez filter

Fig. 2. Noise distributions and the Masreliez nonlinear filter gain function $\partial \ln p(\tilde{y}) / \partial \tilde{y}$.

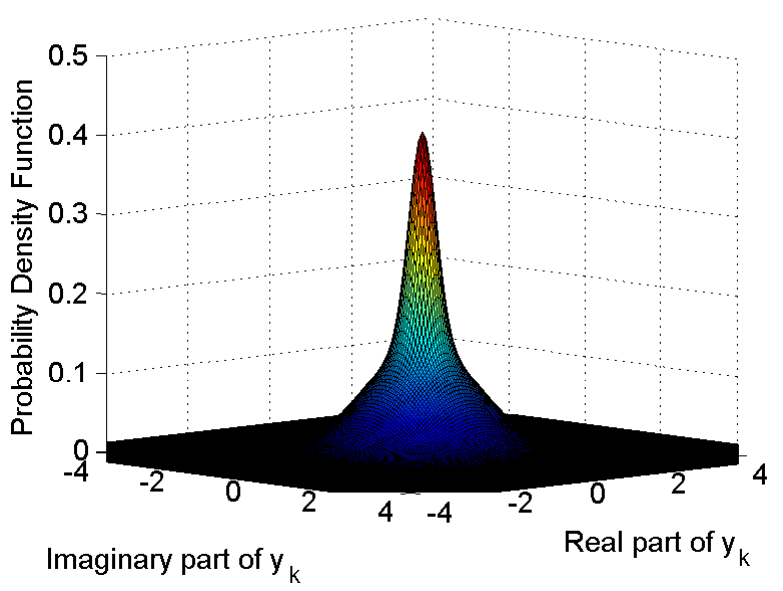

(a) Impulsive noise distributions

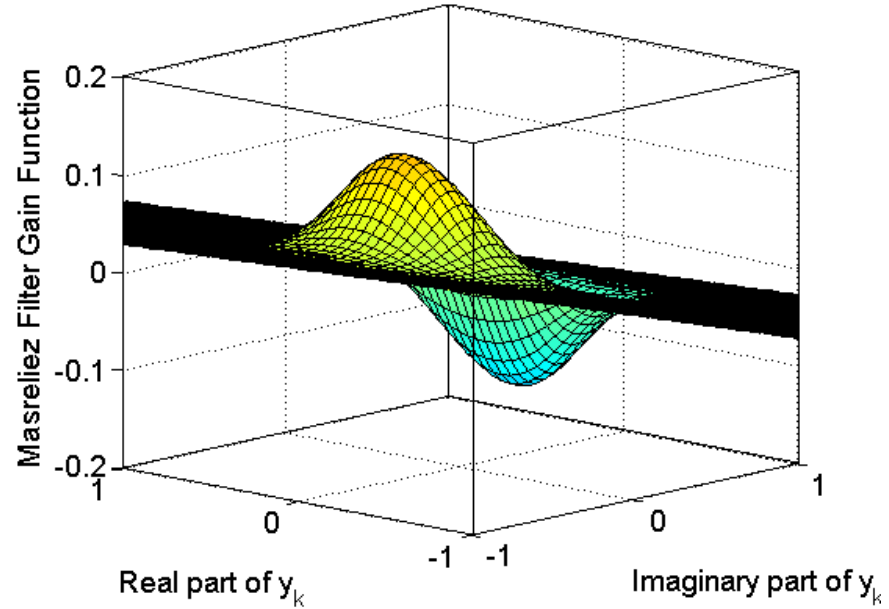

(b) Normalized Masreliez filter

Fig. 3. Complex zero mean impulsive noise distribution and the Masreliez nonlinear filter gain function i.e., $\partial \ln p\left(y_{r}+j y_{i}\right) / \partial\left(y_{r}\right)$.

$$
\Sigma^{-1}=\left(\mathbf{I}+\mathbf{V}^{-1} \mathbf{C}\right)^{-1} \mathbf{V}^{-1} \simeq\left(\mathbf{I}-\mathbf{V}^{-1} \mathbf{C}\right) \mathbf{V}^{-1} .
$$

The parameter $\alpha$ is added here so that the matrix $\mathbf{V}$ is invertible, and its value is chosen to ensure an accurate approximation. Based on our assumption that the matrix $\mathbf{H H}^{*}$ is diagonally dominant, the inverse matrix in (23) can be written as

$$
\Sigma^{-1}=q_{0} \mathbf{I}+q_{1} \mathbf{J}_{1}+q_{2} \mathbf{J}_{2},
$$

where $q_{0}=\frac{2}{\alpha}-\frac{\sigma_{x}^{2}}{\alpha^{2}}, q_{1}=-\frac{\sigma_{1}^{2}}{\alpha^{2}}, q_{2}=\left(\frac{-\sigma_{2}^{2}}{\alpha\left(\alpha+\sigma_{2}^{2}\right)}+\frac{-\sigma_{x}^{2}+\alpha}{\left(\alpha+\sigma_{2}^{2}\right)^{2}}\right)$.

So far we assumed that each received sample has a known noise variance and its state $w_{i}$ is known. To overcome the restriction, we use the law of total expectation to get

$$
\hat{\mathbf{x}}=E_{W}\left[E_{X}[\mathcal{X} \mid \tilde{\mathcal{Y}}=\tilde{\mathbf{y}}, W=\mathbf{w}]\right]=\sum_{\mathbf{w}} p(\mathbf{w} \mid \tilde{\mathbf{y}}) \hat{x}_{\mid W}
$$

Therefore, using the Bayes' law we obtain

$$
\hat{\mathbf{x}}=\mathbf{U}^{*} \Sigma_{\tilde{x}} \mathbf{H}^{*} \prod_{i=1}^{N} \sum_{w} \frac{p\left(y_{i} \mid w_{i}\right) p\left(w_{i}\right)}{\sum p\left(y_{i} \mid w_{i}\right) p\left(w_{i}\right)} \Sigma^{-1} \tilde{\mathbf{y}} .
$$

We again assume that only diagonal elements of covariance matrix $\Sigma$ are related to each $w_{i}$; Hence, on row $i$ the product reduces to one term. We summarize our filter in a form that mimics the LMMSE filter as follows

$$
\hat{\mathbf{x}}^{\text {Masreliez }}=\mathbf{U}^{*} \mathbf{K}^{\text {Masreliez }}(\tilde{\mathbf{y}}) \tilde{\mathbf{y}} .
$$

The Masreliez filter for the approximated solution is defined as

$$
\mathbf{K}^{\text {Masreliez }}(\tilde{\mathbf{y}})=\sigma_{\tilde{x}}^{2} \mathbf{H}^{*}\left(\boldsymbol{\Sigma}_{\mathbf{w}=\mathbf{1}}^{-1} \mathbf{P}_{1}(\tilde{\mathbf{y}})+\boldsymbol{\Sigma}_{\mathbf{w}=\mathbf{2}}^{-1} \mathbf{P}_{2}(\tilde{\mathbf{y}})\right)
$$


where $\Sigma_{\mathbf{w}=\mathbf{k}}$ is the covariance matrix $\Sigma$ once all the states are set to $k$, and matrices $\mathbf{P}_{k}$ 's are defined as

$$
\mathbf{P}_{k}=\operatorname{diag}\left[\frac{p\left(w_{i}=k\right) p\left(y_{i} \mid w_{i}=k\right)}{\sum p\left(w_{i}\right) p\left(y_{i} \mid w_{i}\right)}\right],
$$

and

$$
p\left(y_{i} \mid w_{i}=k\right)=\left(\frac{1}{\sigma_{x}^{2}}+\sigma_{k}^{2}\right)^{-1} \exp \left(-\tilde{y}_{i}^{*}\left(q_{0}+q_{k}\right) \tilde{y}_{i}\right) .
$$

For brevity, we have expressed (28) for $k=1,2$. The formulation can be generalized for higher order of $k$. In (28) the inverse matrices can be calculated off-line. We note that the Masreliez filter given here is a time domain filter, and its coefficient matrix $\mathbf{K}^{\text {Masreliez }}$, unlike the LMMSE filter, is a nonlinear filter of the received signal $\tilde{\mathbf{y}}$.

\section{Simulations And Discussions}

In this section, we provide some simulation results for the proposed VOFDM system with Masreliez filtering in a wide range of impulsive noise environments. There are several power line channel models available in the literature. In this paper we adopt the model introduced in [29], based on practical field measurements. The characteristics of this model can be represented by

$$
H(f)=\sum_{b=0}^{B}\left|\theta_{b}\right| \exp \left(\frac{-2 \pi f d_{b}}{\nu}\right) \exp \left(-\left(\alpha_{0}+\alpha_{1}\right) f^{K} d_{b}\right),
$$

where $B$ is the number of paths, $\theta_{b}<1$ is a weighting factor representing the product of the reflection and transmission factors along the $b^{t h}$ path, $d_{b}=c / \sqrt{\epsilon_{r}}$ is the length of the path (in meters), $c$ is the speed of light and $\epsilon_{r}$ is the dielectric constant of the power line cable. The parameters $\alpha_{0}, \alpha_{1}$ and $K$ are related to the frequency- and distance-dependent attenuation. The time-domain impulse response of the PLC channel can be given by

$$
q(t)=\sum_{b=1}^{B} \beta_{b} \delta\left(t-\tau_{b}\right)
$$

where $\beta_{b}$ is the amplitude of arrivals and $\tau_{b}$ is the arrival times of the multi-path signals.

Impulsive noise is modeled in this study using Middleton Class-A noise model [13], [14]. This model considers the background and impulsive components as a sequence of independent identically distributed random complex memory less variables. The probability density function (pdf) of the complex Class-A noise is given by

$$
f_{\Omega}(n)=\sum_{m=0}^{\infty} \frac{\alpha_{m}}{2 \pi \sigma_{m}^{2}} \exp \left(-\frac{\|n\|^{2}}{2 \sigma_{m}^{2}}\right),
$$

where $\alpha_{m}$ and $\sigma_{m}^{2}$ are given, respectively, as

$$
\alpha_{m}=\frac{e^{-A} A^{m}}{m !},
$$

and

$$
\sigma_{m}^{2}=\sigma^{2}\left(\frac{m / A+\Gamma}{1+\Gamma}\right) .
$$

where $\sigma^{2}$ is the variance of the total noise, $m$ denotes the channel state $(0,1,2, \ldots)$ and $\sigma_{m}^{2}$ represents the noise variance for channel state $m$. The parameter $A$ is referred to as the impulsive index and $\Gamma$ is the Gaussian-to-impulse noise power ratio. There are three parameters specifying the statistical characteristics of this model, namely: $A, \Gamma$ and $\sigma^{2}$. When $A$ is increased, the noise will be similar to a Gaussian noise; reduced values of $A$ mean high noise pulses. In order to visualize this, we now plot in Fig. 4 some numerical results of (33) with different values of $A$ and $\Gamma$. It is visible that when $\Gamma$ is kept constant at 0.001 and the impulsive index is varied from a large value to a small value, two main cases can be highlighted. Firstly, when $A$ is large, the characteristic distribution of the noise is very similar to Gaussian distribution, see Fig. 4a. Secondly, when $A$ becomes small, the distribution shows very impulsive characteristics and therefore the performance is expected to degrade. Similarly, same observations can be noticed from Fig. $4 \mathrm{~b}$ when $\Gamma$ is changed while keeping $A$ constant.

To evaluate the equalizer filters for VOFDM system, we tested the algorithm with Middleton Class-A noise where two terms were considered in generating the noise. For LMMSE filter the received signal is divided into $M \times L$ matrix and each row is fed into the DFT module. The results are accumulated into an $M \times L$ matrix, where each column goes through the LMMSE filter introduced in (10). The decisions are made by the detector given in (14). However, for the VOFDM system, after removing the $\mathrm{CP}$, the received signal is equalized using (28). Similar to the LMMSE filter, the equalized signal goes through the DFT modules and the detector. The simulation parameters used here are: $N=256$ sub-carrier, base-band modulation is QPSK with gray mapping and the number channel taps is 4 . Recall that $M$ is the size of each vector in (2). To show the effect of the vector block size of the proposed VOFDM system performance, we plot in Fig. 5 the BER performance versus SNR for $M=1,2,4,8$, and 16 when $A=0.1$ and $\Gamma=0.01$. The curve for $M=1$ indicates the OFDM system. For the PLC channel, we use reflection factor $K=1, \alpha_{0}=0, \alpha_{1}=7.8 \times 10^{-10} \mathrm{~S} / \mathrm{m}$ and delay $\tau_{b}=d_{z} / \nu$ where $\nu=1.5 \times 10^{8} \mathrm{~m} / \mathrm{s}$ [29]. It was also shown in [29] that five paths are enough to accurately model the impulse response of the channel. It is clear that the proposed system always outperforms the conventional OFDM system (i.e., $M=1$ ). It can also be observed that this improvement becomes larger as we increase the number of the vector blocks. Additionally, increasing the SNR minimizes the BER irrespective of the value of $M$. It is worth pointing out that the computation time of the proposed method is reasonably small compared to that of the ML detector, hence it can be applied in both realtime as well as off-line analyses. Specifically, the complexity of equalization is at most $O\left(N^{2}\right)$, and this may grow to $O\left(N^{3}\right)$ for online calculations. Note that there are several methods that have been reported in the literature to further reduce computation complexity of similar systems, see e.g. [30]. 


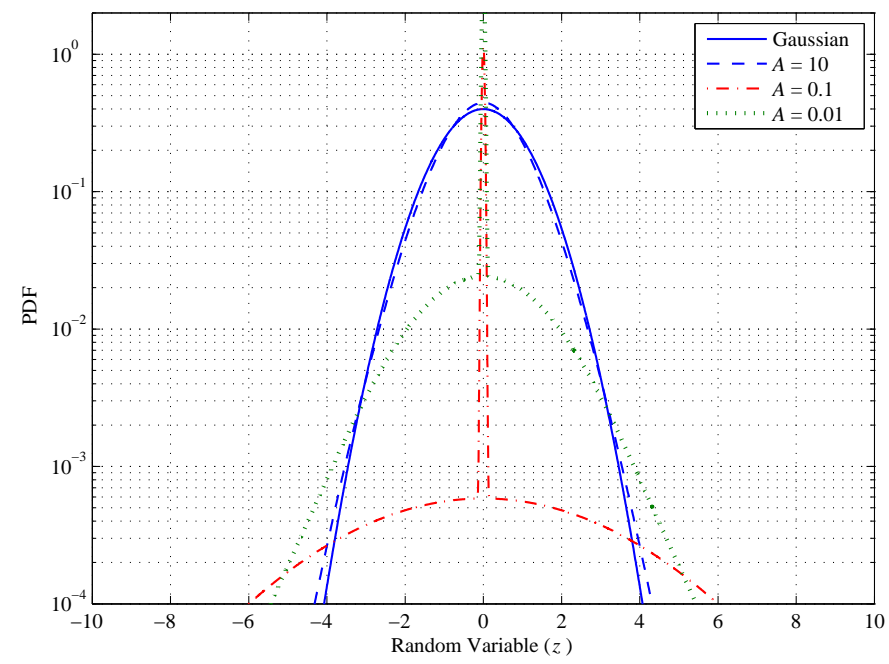

(a) Middleton Class-A pdfs for different values of $A$ when $\Gamma=0.001$.

Fig. 4. The effect of Middleton Class-A noise model parameters on its pdf.

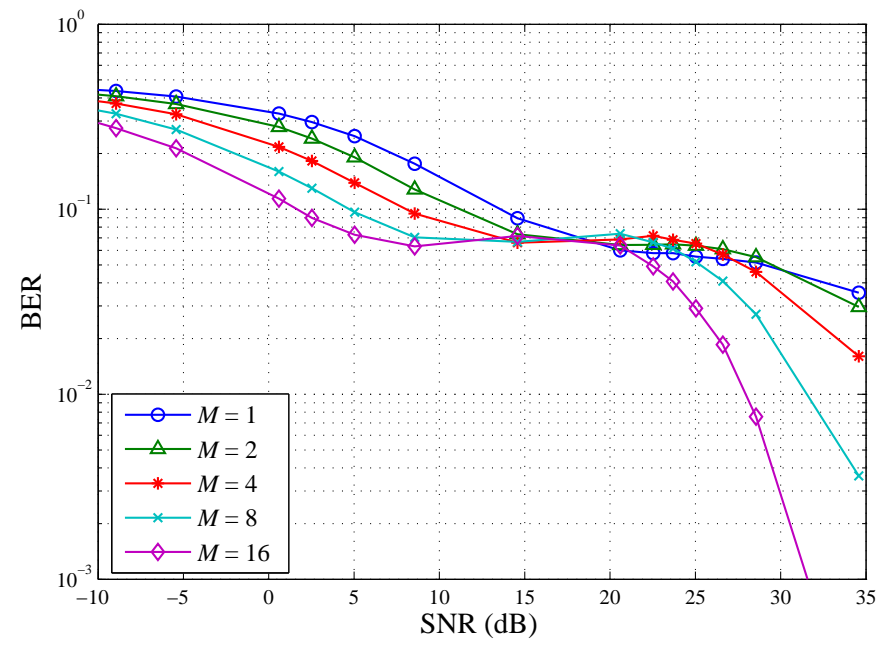

Fig. 5. BER performance of the proposed VOFDM system for various values of $M$ when $A=0.1$ and $\Gamma=0.01$.

Now, to illustrate the impact of the noise parameter $\Gamma$ on the VOFDM system performance, we illustrate the BER performance in Fig. 6 for a wide range of $\Gamma$ values while keeping $A=0.1$ and $M=16$. The first observation one can see from these results is that increasing the SNR will always offer improved BER performance for all the $\Gamma$ values under consideration. For $\Gamma=1$, the Middleton Class-A noise density is similar to Gaussian and Masreliez equalizer performs the same as LMMSE. In addition, it is clear that as $\Gamma$ decreases, i.e. the impulsive noise power becomes higher relative to the Gaussian one, the performance degrades significantly, and the gain from Masreliez equalizer increases. At higher SNR's the performance of LMMSE and Masreliez equalizer converges; This is due to small magnitude of spikes in impulsive noise that filter can not distinguish from the transmitted data.

When there is no ISI in the channel, we are able to calculate the error bounds for the detector. For the noise model given

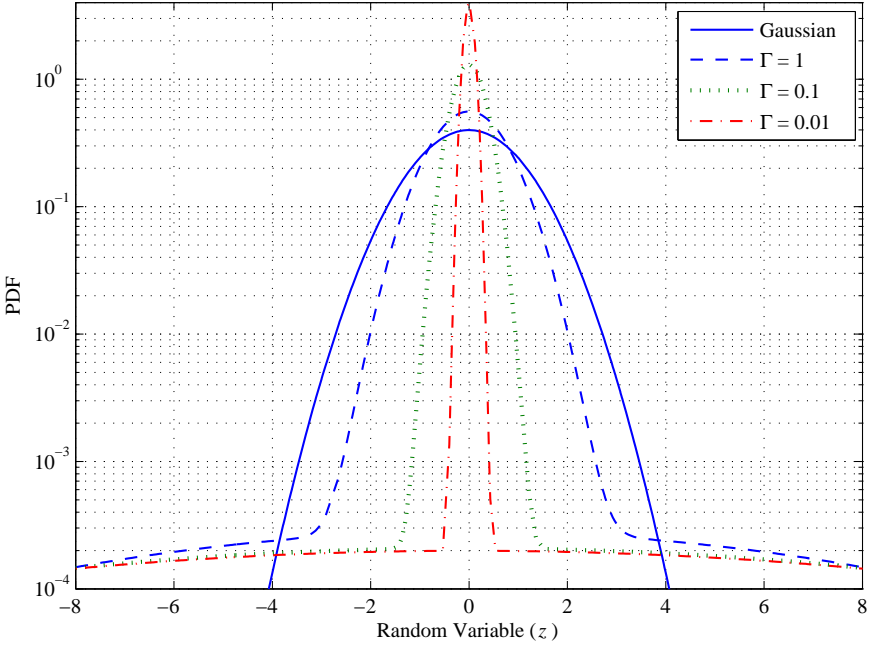

(b) Middleton Class-A pdfs for different values of $\Gamma$ when $A=0.01$.

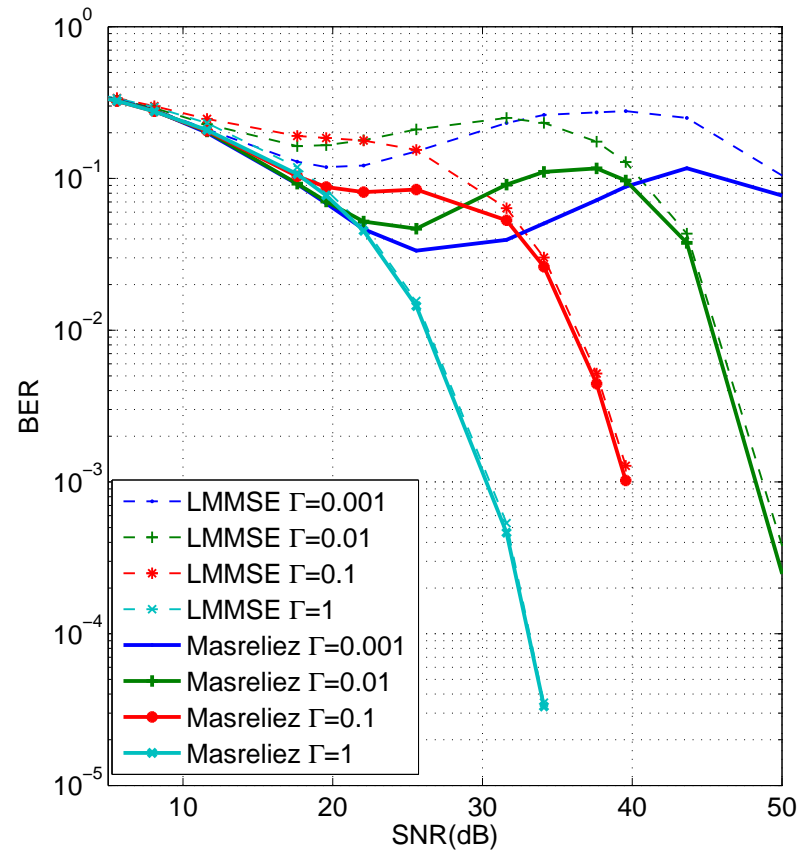

Fig. 6. BER performance versus SNR for the proposed VOFDM system with different values of $\Gamma$ when $A=0.05$ and $M=16$.

in (33), we can write a bound on the probability of error as follows [31]

$$
P_{e}=\sum_{m=0}^{\infty} \alpha_{m} P_{e}\left(\sigma_{m}\right),
$$

where $P_{e}\left(\sigma_{m}\right)$ is the probability of error for uncoded transmission in the presence of the complex additive white Gaussian noise (CAWGN) with variance $\sigma_{m}^{2}$. Fig. 7 depicts the BER for different values of $A$ with $\Gamma=0.01$ and $M=16$ for the BPSK modulation over a memoryless channel. It is interesting to see 


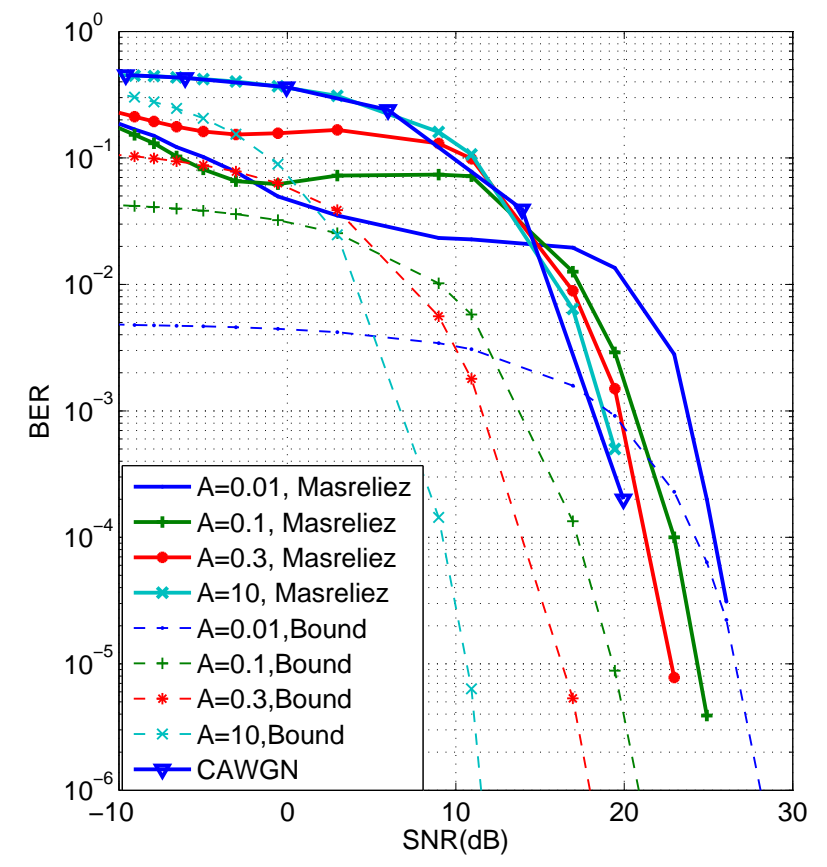

Fig. 7. BER performance versus SNR for the proposed VOFDM system with several values of $A$ when $\Gamma=0.01$ and $M=16$.

that the results on this figure can be divided into two SNR regions over which the BER behaves differently with respect to $A$. These regions can be classified as low SNR $(\mathrm{SNR} \lesssim 20 \mathrm{~dB})$ and high $\mathrm{SNR}(\mathrm{SNR} \gtrsim 20 \mathrm{~dB})$. In the first region, it is clear that as the value of $A$ decrease, i.e. noise becomes more impulsive, the performance improves whereas the opposite is true in the second SNR region. As $A$ increases, the noise characteristics becomes close to CAWGN. For CAWGN the symbol-by-symbol detector is optimal. We have compared our Masreliez equalizer for $A=10$ to the CAWGN symbol-bysymbol detector to validate our detection strategy.

\section{CONCLUSiON}

In this paper, we addressed the transmission over PLC channels using a VOFDM system in conjunction with the Masreliez filter for non-Gaussian noise. We derived a nonlinear equalizer to combat the damaging effect of impulsive noise present in PLC. We implemented the Masreliez filter in a wide range of impulsive noise environments ranging from weakly- to heavily-disturbed environments. System performance was evaluated in terms of the BER. The results have shown that considerable improvements can be attained with the proposed system relative to the conventional OFDM system and this improvement becomes higher as we increase the size of vector blocks. We compared the proposed equalizer for different set of parameters of Middleton Class-A noise with LMMSE equalizer. The performance analysis shows that the proposed Masreliez filter is effective in low SNR where the spikes of impulsive noise can be distinguished from the signal. The improvement was compared in a wide range of Middleton Class-A noise parameters.

\section{APPENDIX A}

DERIVATION OF MASRELiEz Filter IN (17)

The estimate of $\mathbf{x}$ is given as conditional expectation of transmitted signal given received signal

$$
\hat{\mathbf{x}}=\mathbb{E}[\mathcal{X} \mid \tilde{\mathcal{Y}}=\tilde{\mathbf{y}}]
$$

However, using the linearity of expectation we can write the estimator in time domain as [20]

$$
\hat{\mathbf{x}}=\mathbf{U}^{*} \mathbb{E}[\mathbf{U} \mathcal{X} \mid \mathcal{Y}=\tilde{\mathbf{y}}]=\mathbf{U}^{*} \int \tilde{\mathbf{x}} p(\tilde{\mathbf{x}} \mid \tilde{\mathbf{y}}) d \tilde{\mathbf{x}}_{i} d \tilde{\mathbf{x}}_{r},
$$

where subscript $r$ and $i$ represent the real and imaginary parts of the variable, respectively. By Bayes' law we can write (38) in terms of noise distribution $p_{\boldsymbol{\Omega}}$

$$
\hat{\mathbf{x}}=\frac{1}{p(\tilde{\mathbf{y}})} \mathbf{U}^{*} \int \tilde{\mathbf{x}} p_{\boldsymbol{\Omega}}(\tilde{\mathbf{y}} \mid \tilde{\mathbf{x}}) p(\tilde{\mathbf{x}}) d \tilde{\mathbf{x}}_{i} d \tilde{\mathbf{x}}_{r} .
$$

Now, suppose $\tilde{\mathbf{x}}$ is a vector of circularly symmetric Gaussian random variables, $\mathcal{C N}\left(\mathbf{0}, \boldsymbol{\Sigma}_{\tilde{x}}\right)$, multiplied by covariance of $\tilde{\mathbf{x}}$ and its inverse we get

$$
\hat{\mathbf{x}}=\frac{1}{p(\tilde{\mathbf{y}})} \mathbf{U}^{*} \boldsymbol{\Sigma}_{\tilde{x}} \int p_{\boldsymbol{\Omega}}(\tilde{\mathbf{y}} \mid \tilde{\mathbf{x}}) \boldsymbol{\Sigma}_{\tilde{x}}^{-1} \tilde{\mathbf{x}} p(\tilde{\mathbf{x}}) d \tilde{\mathbf{x}}_{i} d \tilde{\mathbf{x}}_{r}
$$

which, using Wirtinger derivatives [26], can be further simplified as

$$
\hat{\mathbf{x}}=-\frac{1}{p(\tilde{\mathbf{y}})} \mathbf{U}^{*} \boldsymbol{\Sigma}_{\tilde{x}} \int p_{\boldsymbol{\Omega}}(\tilde{\mathbf{y}} \mid \tilde{\mathbf{x}}) \frac{\partial}{\partial \tilde{\mathbf{x}}^{*}} p(\tilde{\mathbf{x}}) d \tilde{\mathbf{x}}_{i} d \tilde{\mathbf{x}}_{r}
$$

Then, integrating by parts we have

$$
\hat{\mathbf{x}}=\frac{1}{p(\tilde{\mathbf{y}})} \mathbf{U}^{*} \boldsymbol{\Sigma}_{\tilde{x}} \int \frac{\partial}{\partial \tilde{\mathbf{x}}^{*}} p_{\boldsymbol{\Omega}}(\tilde{\mathbf{y}} \mid \tilde{\mathbf{x}}) p(\tilde{\mathbf{x}}) d \tilde{\mathbf{x}}_{i} d \tilde{\mathbf{x}}_{r} .
$$

Finally, using chain rule we take the derivative with respect to $\tilde{\mathbf{y}}$ to get

$$
\hat{\mathbf{x}}=\frac{1}{p(\tilde{\mathbf{y}})} \mathbf{U}^{*} \boldsymbol{\Sigma}_{\tilde{x}} \mathbf{H}^{*} \frac{\partial}{\partial \tilde{\mathbf{y}}} \int p_{\boldsymbol{\Omega}}(\tilde{\mathbf{y}} \mid \tilde{\mathbf{x}}) p(\tilde{\mathbf{x}}) d \tilde{\mathbf{x}}_{i} d \tilde{\mathbf{x}}_{r}
$$

where we changed the order of integration and derivation. Equation (42) can be written more succinctly as in (17).

\section{REFERENCES}

[1] S. Galli, A. Scaglione, and Z. Wang, "For the grid and through the grid: The role of power line communications in the smart grid," Proc. IEEE, vol. 99, no. 6, pp. 998-1027, Jun. 2011.

[2] N. Shlezinger and R. Dabora, "On the capacity of narrowband PLC channels," IEEE Trans. Commun., vol. 63, no. 4, pp. 1191-1201, Apr. 2015.

[3] K. Rabie and E. Alsusa, "Effective noise cancellation using single-carrier FDMA transmission in power-line channels," IEEE Trans. Power Del., vol. 29, no. 5, pp. 2110-2117, Oct. 2014.

[4] R. Pighi, M. Franceschini, G. Ferrari, and R. Raheli, "Fundamental performance limits of communications systems impaired by impulse noise," IEEE Trans. Commun., vol. 57, no. 1, pp. 171-182, Jan. 2009.

[5] D. Middleton, "Canonical and quasi-canonical probability models of class A interference," IEEE Trans. Electromagn. Compat., vol. EMC25, no. 2, pp. 76-106, May 1983.

[6] — "Non-gaussian noise models in signal processing for telecommunications: new methods an results for class-A and class-B noise models," IEEE Trans. Inf. Theory, vol. 45, no. 4, pp. 1129-1149, May 1999. 
[7] P. Amirshahi, S. Navidpour, and M. Kavehrad, "Performance analysis of uncoded and coded OFDM broadband transmission over low voltage power-line channels with impulsive noise," IEEE Trans. Power Del., vol. 21, no. 4, pp. 1927-1934, Oct. 2006.

[8] V. C. Gungor, D. Sahin, T. Kocak, S. Ergut, C. Buccella, C. Cecati, and G. P. Hancke, "Smart grid technologies: communication technologies and standards," IEEE Trans. on Ind. inform., vol. 7, no. 4, pp. 529-539, Sept. 2011.

[9] G. Bumiller, L. Lampe, and H. Hrasnica, "Power line communication networks for large-scale control and automation systems," IEEE Commun. Mag., vol. 48, no. 4, pp. 106-113, Jun. 2010.

[10] S. Galli, A. Scaglione, and Z. Wang, "For the grid and through the grid: The role of power line communications in the smart grid," Proc. IEEE, vol. 99, no. 6, pp. 998-1027, Jun. 2011.

[11] M. Lee, R. E. Newman, H. A. Latchman, S. Katar, and L. Yonge, "HomePlug 1.0 powerline communication LANsâATTprotocol description and performance results," Int. J. Commun. Syst., vol. 16, no. 5, pp. 447473, Jun. 2003.

[12] K. Saaifan and W. Henkel, "Decision boundary evaluation of optimum and suboptimum detectors in class-A interference," IEEE Trans. Commun., vol. 61, no. 1, pp. 197-205, Jan. 2013.

[13] K. Rabie and E. Alsusa, "Preprocessing-based impulsive noise reduction for power-line communications," IEEE Trans. Power Del., vol. 29, no. 4, pp. 1648-1658, Aug. 2014.

[14] S. Zhidkov, "Analysis and comparison of several simple impulsive noise mitigation schemes for OFDM receivers," IEEE Trans. Commun., vol. 56, no. 1, pp. 5-9, Jan. 2008.

[15] Y. Li, I. Ngebani, X.-G. Xia, and A. Host-Madsen, "On performance of vector OFDM with linear receivers," IEEE Trans. Signal Process., vol. 60, no. 10, pp. 5268-5280, Oct. 2012.

[16] P. Cheng, M. Tao, Y. Xiao, and W. Zhang, "V-OFDM: On performance limits over multi-path rayleigh fading channels," IEEE Trans. Commun., vol. 59, no. 7, pp. 1878-1892, May 2011.

[17] X. Xia, "Precoded and vector OFDM robust to channel spectral nulls and with reduced cyclic prefix length in single transmit antenna systems," IEEE Trans. Commun., vol. 49, no. 8, pp. 828-838, Aug. 2001.

[18] H. Zhang, X.-G. Xia, L. J. Cimini, and P. C. Ching, "Synchronization techniques and guard-band-configuration scheme for single-antenna vector-OFDM systems," IEEE Trans. Wireless Commun., vol. 4, no. 5, pp. 2454-2464, Sept. 2005.

[19] C. Masreliez, "Approximate non-gaussian filtering with linear state and observation relations," IEEE Trans. Autom. Control, vol. 20, no. 1, pp. 107-110, Feb. 1975.

[20] X. Wang and H. Poor, "Joint channel estimation and symbol detection in Rayleigh flat-fading channels with impulsive noise," IEEE Commun. Lett., vol. 1, no. 1, pp. 19-21, Jan. 1997.

[21] A. J. Laub, Matrix Analysis for Scientists and Engineers. Slam, 2005.

[22] G. H. Golub and C. F. V. Loan., Matrix Computations. Baltimore, MD: Johns Hopkins Univ. Press, 1983.

[23] Q. Guo and L. Ping, "LMMSE turbo equalization based on factor graphs," IEEE J. Sel. Areas in Commun., vol. 26, no. 2, pp. 311-319, Feb. 2008.

[24] J. G. Proakis, Digitial Communications. 3rd ed. New York: McGraw Hill, 1995.

[25] M. Tuchler, A. Singer, and R. Koetter, "Minimum mean squared error equalization using a priori information," IEEE Trans. Signal Process., vol. 50, no. 3, pp. 673-683, Mar. 2002.

[26] L. Rusch and H. Poor, "Narrowband interference suppression in CDMA spread spectrum communications," IEEE Trans. Commun., vol. 42, no. 234, pp. 1969-1979, Feb. 1994.

[27] Z.-Z. Bai, G. H. Golub, and M. K. Ng, "Hermitian and skew-Hermitian splitting methods for non-Hermitian positive definite linear systems," SIAM Journ. Matrix Anal. Appl., vol. 24, no. 3, pp. 603-626, Jul. 2003.

[28] G. Ndo, F. Labeau, and M. Kassouf, "A Markov-Middleton model for bursty impulsive noise: Modeling and receiver design," IEEE Trans. Power Del., vol. 28, no. 4, pp. 2317-2325, Oct. 2013.

[29] B. Adebisi, Broadband Transmission over Indoor Power-Line channels. $\mathrm{Ph} . \mathrm{D}$. dissertation, School of Computing and Communications: Lancaster University, UK, 2009.

[30] M. Noh, Y. Lee, and H. Park, "Low complexity LMMSE channel estimation for OFDM," IEE Proc. Commun., vol. 153, no. 5, pp. 645650, Oct. 2006.

[31] R. Haring and A. J. H. Vinck, "Performance bounds for optimum and suboptimum reception under Class-A impulsive noise," IEEE Trans. Commun., vol. 50, no. 7, pp. 1130-1136, Jul 2002.

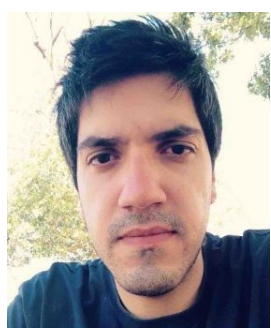

Cinna Soltanpur received his M.Sc. degree in communication systems from Lancaster University, Lancaster, UK in 2011 and his Ph.D. in electrical and computer engineering from The University of Oklahoma, OK, USA in 2016 . He is currently with Viavi Solutions and his research interests are in information theory, signal processing, storage devices and smart grids.

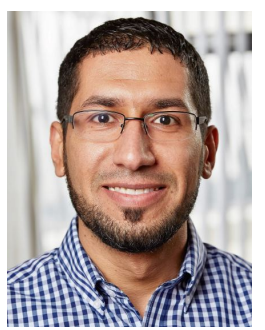

Khaled Maaiuf Rabie (SM'12, M'15) received the B.Sc. degree (with Hons.) from University of Tripoli, Libya, and the M.Sc. degree (with Hons.) from the university of Manchester, UK, in 2008 and 2010 , respectively. In 2011, he joined the university of Manchester where he worked as part-time staff and received his Ph.D. degree in Communication Engineering in 2015. He is currently a Postdoctoral Research Associate at Manchester Metropolitan University (MMU), Manchester, UK. His research interests include signal processing and analysis of powerline and wireless communication networks. Dr. Rabie received several awards, both nationally and internationally, including the Agilent Technologies' best M.Sc. student award, the Manchester Doctoral College Ph.D. scholarship and the MMU Outstanding Knowledge Exchange Project award of 2016. He was also the recipient of the best student paper award at the IEEE International Symposium on Power Line Communications and its applications (ISPLC) in 2015, Texas, US

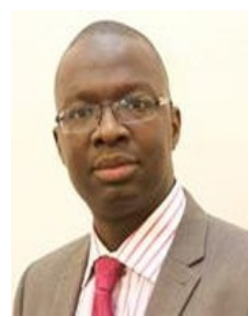

Bamidele Adebisi (M'06, SM'15) received his Master's degree in advanced mobile communication engineering and Ph.D. in communication systems from Lancaster University, UK, in 2003 and 2009, respectively. Before that, he obtained a Bachelor's degree in electrical engineering from Ahmadu Bello University, Zaria, Nigeria, in 1999. He was a senior research associate in the School of Computing and Communication, Lancaster University between 2005 and 2012. He joined Metropolitan University, Manchester in 2012 where he is currently a Reader in Electrical and Electronic Engineering. He has worked on several commercial and government projects focusing on various aspects of wireline and wireless communications. He is particularly interested in Research and Development of communication technologies for electrical energy monitoring/management, transport, water, critical infrastructures protection, home automation, IoTs and Cyber Physical Systems. He has several publications and a patent in the research area of data communications over power line networks and smart grid. He is a member of IET and a senior member of IEEE.

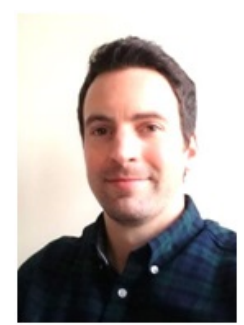

Andrew Wells received his Master's degree in mobile communications and Ph.D. in communications system and computer science from Lancaster University, UK in 2007 and 2010, respectively. Following this, in 2011 he moved to the advanced electrical research department at Jaguar Land Rover working on several projects focussing on the development of future infotainment and vehicle connectivity. He joined the Electrical and Electronic Software Engineering department at JLR and is the technical lead for JLRs cross car-line next generation audio platform. $\mathrm{He}$ is particularly interested in the research and development of communications technologies for vehicle communications (V-X), IoTs, autonomous vehicles and automotive security. 